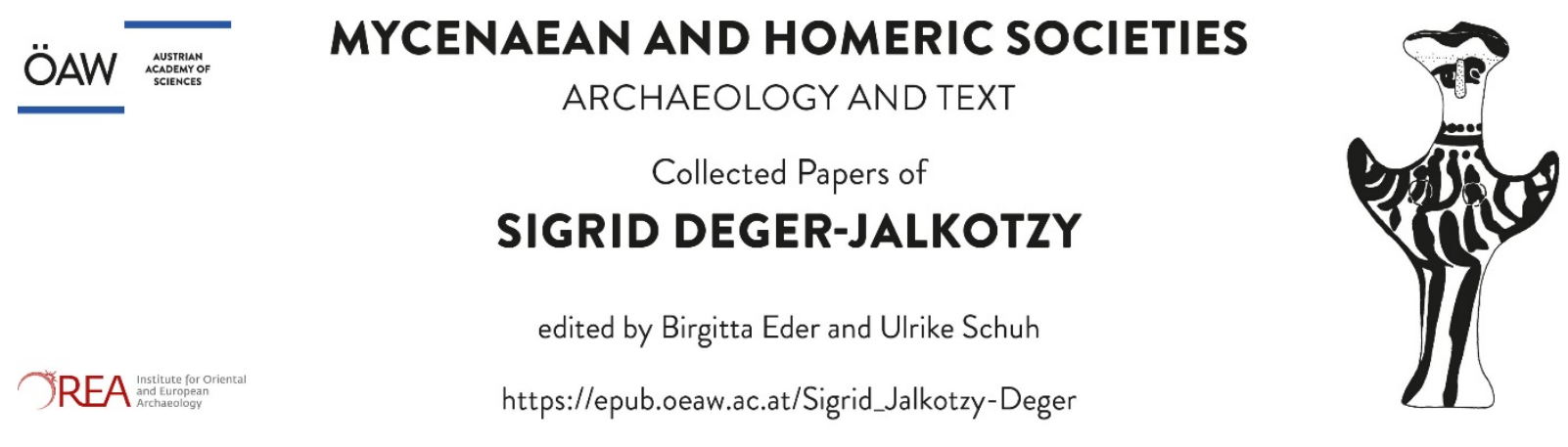

31.

S. Deger-Jalkotzy - W.-D. Niemeier

\title{
Die mykenische Koiné
}

in: Der Neue Pauly. Enzyklopädie der Antike 1 (Stuttgart - Weimar 1996) 150-156

(C) J.B. Metzler [Brill] https://referenceworks.brillonline.com/browse/brill-s-new-pauly mit freundlicher Genehmigung / with kind permission

Dieses Dokument darf ausschließlich für wissenschaftliche Zwecke genutzt werden (Lizenz CC BY-NC-ND), gewerbliche Nutzung wird urheberrechtlich verfolgt.

This document is for scientific use only (license CC BY-NC-ND), commercial use of copyrighted material will be prosecuted. 


\section{DER NEUE PAULY}

\section{Enzyklopädie der Antike}

Herausgegeben

von Hubert Cancik und

Helmuth Schneider
Altertum

Band I A-Ari 


\section{DER NEUE PAULY}

\section{(DNP)}

\section{Fachgebietsherausgeber}

Prof. Dr. Gerhard Binder, Bochum

Kulturgeschichte

Prof. Dr. Hubert Cancik, Tübingen

Geschäftsfuihrender Herausgeber

Prof. Dr. Walter Eder, Bochum

Alte Geschichte, Rezeption: Staatstheorie, Politik

Prof. Dr. Burkhard Fehr, Hamburg

Archäologie, Rezeption: Kunst, Architektur,

Alltagsleben, Medien

Prof. Dr. Bernhard Forssman, Erlangen

Sprachwissenschaft

Prof. Dr. Fritz Graf, Basel

Religion und Mythologie, Rezeption: Religion

PD Dr. Hans Christian Günther, Freiburg

Textwissenschaft

Dr. Christoph Höcker, Hamburg

Archäologie, Rezeption: Kunst, Architektur,

Alltagsleben, Medien

Prof. Dr. Christian Hünemörder, Hamburg

Naturwissenschaften und Technik

Dr. Margarita Kranz, Berlin

Rezeption: Philosophie

Prof. Dr. André Laks, Lille

Philosophie

Prof. Dr. Manfred Landfester, Gießen, Rezeption: Wissenschaftsgeschichte, geschäftsführender Herausgeber: Rezeptions- und Wissenschaftsgeschichte
Prof. Dr. Maria Moog-Grünewald, Tübingen Rezeption: Komparatistik und Literatur

Prof. Dr. Dr. Glenn W. Most, Heidelberg Griechische Philologie

Dr. Johannes Niehoff, Freiburg Judentum, östliches Christentum

Prof. Dr. Hans Jörg Nissen, Berlin Orientalistik

Prof. Dr. Vivian Nutton, London Medizin, Rezeption: Medizin

Prof. Dr. Eckart Olshausen, Stuttgart Historische Geographie

Prof. Dr. Filippo Ranieri, Saarbrücken Rezeption: Rechtsgeschichte

Prof. Dr. Johannes Renger, Berlin Orientalistik

Prof. Dr. Volker Riedel, Jena

Rezeption: Erziehungswesen

Prof. Dr. Jörg Rüpke, Potsdam Lateinische Philologie, Rhetorik

Prof. Dr. Gottfried Schiemann, Tübingen Recht

Prof. Dr. Helmuth Schneider, Kassel Geschäftsfuihrender Herausgeber; Wirtschaftsund Sozialgeschichte

Prof. Dr. Frieder Zaminer, Berlin Musik, Rezeption: Musik

\section{Redaktion}

Beate Baumann

Jochen Derlien

Christa Frateantonio

Dr. Matthias Kopp

Anne-Maria Wittke 


\section{Inhaltsverzeichnis}

Der neue Pauly : Enzyklopädie der Antike / hrsg.

Benutzungshinweise

IX

von Hubert Cancik und Helmuth Schneider. -

Transkriptionstabellen

$\mathrm{X}$

ISBN 3-476-0 I 470-3

NE: Cancik, Hubert [Hrsg.]

Bd. I. A-Ari. - 1996

ISBN 3-476-0I 47 I-I

Abkürzungsverzeichnis

Kartenverzeichnis

XIVIII

Abbildungsverzeichnis

XLIX

Verzeichnis der Autoren

L

Gedruckt auf chlorfrei gebleichtem, säurefreiem und alterungsbeständigem

Verzeichnis der Übersetzer

Papier

Dieses Werk einschließlich aller seiner

Lemmata

Teile ist urheberrechtlich geschützt. Jede Verwertung außerhalb der engen Grenzen des Urheberrechtsgesetzes ist ohne Zustimmung des Verlages unzulässig und strafbar. Das gilt insbesondere für Vervielfältigungen, Übersetzungen, Mikroverfilmungen und die Einspeicherung und Verarbeitung in elektronischen Systemen.

ISBN 3-476-0 I 470-3 (Gesamtwerk)

ISBN 3-476-0I47I-I (Band I A-Ari)

(C) 1996 J.B. Metzlersche Verlagsbuchhandlung und Carl Ernst Poeschel Verlag $\mathrm{GmbH}$ in Stuttgart

Typographie und Ausstattung: Brigitte und Hans Peter Willberg Grafik und Typographie der Karten: Richard Szydlak

Abbildungen: Günter Müller Satz: pagina $\mathrm{GmbH}$, Tübingen Gesamtfertigung: Franz Spiegel Buch GmbH, Ulm

Printed in Germany

Verlag J. B. Metzler Stuttgart · Weimar 
keinen Bruch in der Entwicklung. Es wurden die Neuen Paläste erbaut, und einige Indizien sprechen dafür, daß die gesamte Insel nun unter der Kontrolle von Knossos stand [31]. Die Indizien für min. Präsenz und min. Einfluß im ägäischen Raum sind in der Neupalastzeit noch stärker und weiter verbreitet als in der Altpalastzeit, und dieses Phänomen ist wiederum mit der Rolle des Handels und der Suche nach Metall zu verbinden. Der Charakter der min. Einflüsse zeigenden Siedlung ist in der Forsch. kontrovers diskutiert worden. Die Bandbreite der Vorschläge reicht von ihrer Interpretation als Teile eines min. Seereiches [32] bis jener als unabhängige Handelsstädte, die nur kulturelle Einflüsse aufnahmen [33]. Gegen letztere Deutung spricht aber eine Reihe von Indizien. So wurden in Akrotiri auf Thera, Phylakopi auf Melos, Agia Irini auf Keos, Kastri auf Kythera und in Milet Zeugnisse für den aktiven $\mathrm{Ge}-$ brauch der min. Linear A-Schrift gefunden, an den ersten drei Fundplätzen sogar Tontafeln administrativen Charakters $[34 ; 35]$. Eine ähnliche Verbreitung zeigen Bleigewichte des min. Gewichtssystems. Die tatsächliche Präsenz von Minoern auf einer Reihe von Kykladen- und Dodekanes-Inseln sowie an der Südwestküste Kleinasiens belegt das dortige Auftreten von min. Haushaltsware wie konischen Näpfen, dreibeinigen Kochtöpfen und Webgewichten des scheibenförmigen min. Standard-Typus. Wir haben dabei zw. rein min. Niederlassungen (Kastri auf Kythera, Trianda/lalysos auf Rhodos, Milet) und solchen Siedlungen zu unterscheiden, in denen Einheimische die Mehrheit der Bevölkerung stellten (Akrotiri auf Thera, Phylakopi auf Melos, Agia Irini auf Keos). Die min. Koine in der Ägäis zur Zeit der kret. Neupalastzeit drückt sich auch in der Übernahme min. Vorbilder in der Architektur, in der vor allem rel. Ikonographie der Freskomalerei sowie in Kultobjekten aus. Ein Zeugnis dafür, daß der Einfluß Kretas auf die ägäischen Inseln nicht nur ein kultureller, sondern auch ein polit. war, findet sich in den ägypt. Quellen, die von Kreta und den "Inseln in der Mitte der großen grünen See" als polit. Einheit sprechen [36]. Der arch. Befund und die ägypt. Quellen scheinen damit die histor. Realität der Überlieferung von der "Thalassokratie des Minos" in ägäischen Meer zu bestätigen. Dagegen ist vorgebracht worden, daß diese Legende nicht älter als Herodot sei [37], aber bereits von Hesiod wurde Minos als der königlichste der sterblichen Könige bezeichnet, der über die meisten der ringsum lebenden Menschen herrscht [38]. Zu der "kulturellen" Koine der minoisierten Ägäis gehört seit der Zeit der sog. Schachtgräber von Mykene und der ersten Tholosgräber in Messenien, d.h. seit der Entstehung der myk. Kultur, auch Zentral- und Südgriechenland. Der vorausgehenden mittelhelladischen Epoche war eine künstlerische Tradition fremd, so daß die aufstrebenden festländischen Eliten min. Prestigeobjekte importierten oder min. Stil und min. Ikonographie imitieren ließen [39]. Diese kulturelle Anhängigkeit war aber keineswegs mit einer polit. verbunden. Außerhalb der Ägäis zeigt die neupalastzeitliche Keramik eine ähnliche Verbreitung wie die altpalastzeitliche. Von bes. Interesse sind in ägäischer Freskotechnik, Ikonographie und Stil ausgeführte Wandmalereien der Hyksos-Zeit und frühen XVIII. Dynastie in der Levante (Alalach, Tel Kabri) und Ägypten (Auaris), die nicht ohne die zeitweise Präsenz von ägäischen Künstlern denkbarist [40; 4 I ]. Schließlich bezeugen ägypt. Quellen sowie die Darstellungen von Kretern (Keftiu) in Beamtengräbern der früheren XVIII. Dynastie in ägypt. Theben diplomatische Verbindungen und Handelsbeziehungen zw. Ägypten und Kreta.

\section{B. 4 Die myKenische Koine}

(Ca. 1 400-1 200 v. Chr.). Urspr. als Spezialterminus für die überregionale stilistische Einheitlichkeit myk. Keramikstile des 14.Jh. v. Chr. geprägt (koine st)/le), läßt sich "Koine" darüber hinaus zur Charakterisierung der insgesamt sehr homogenen Kultur der myk. Palastperiode des 14. und 13. Jhs.v. Chr. anwenden. Herausbildung und Verbreitung dieser kulturellen Koine waren eng mit der Entwicklung und Gesch. jenes Herrschaftsund Wirtschaftssystems verbunden, welches sämtliche Funktionen eines Staates in den als "Paläste" bezeichneten architektonischen Großanlagen konzentrierte und welches dem arch. Befund nach um 1400 v. Chr. im myk. Griechenland eingeführt wurde. Die myk. Paläste verdankten ihre Existenz der Herausbildung von Staaten mit größerer Flächenausdehnung, deren monarchisches Regime theokratisch und mit großer Machtfülle ausgestattet war. Zentralistische Herrschaft und "redistributives" Wirtschaftssystem erforderten eine komplizierte, von einem umfangreichen Beamtenapparat getragene Administration und eine Kontrolle durch Bürokratie. Ergebnis war die Entwicklung der sog. $\rightarrow$ Linear B-Schrift, deren auf Tontäfelchen aufgezeichnete Texte grundlegende Quellen für die Erforsch. der myk. Palastära sind. Durch sie wird eine erste, wichtige Facette der myk. Koine offenbar: Anders als die min. Koine, wurde sie getragen von Sprechern des Griechischen.

Welche polit. und ökonomischen Prozesse zur Herausbildung und Etablierung myk. Paläste in späten 15. oder frühen 14.Jh. v. Chr. führten, ist wenig klar. Arch. Hinweise deuten auf Machtausdehnung auf Kosten kleinerer Herrschaftsbereiche, die in den Territorien der Palast-Staaten aufgingen. Einige Faktoren sind einer endogenen Entwicklung aus der frühmyk. Tradition zuzuschreiben, andere (z.B. der extreme Zentralismus von Herrschaft tind Verwaltung) eher oriental. Vorbildern. Offenkundig ist die Vorbildwirkung der min. Paläste für Funktionspläne, Architektur, Ausstattung und für die Grundzüge der Verwaltung myk. Paläste. Die myk. Linear B-Schrift wurde auf der Basis der nicht-griech. min. Kanzleischrift (Linear A) entwickelt, und dasselbe gilt für das myk. Maß- und Zahlensystem. Viele Forscher rechnen auch mit einem min. Vorbild für die myk. Königsideologie. Möglicherweise hing diese Übernahme min. Elemente mit polit. Ereignissen gegen Ende 


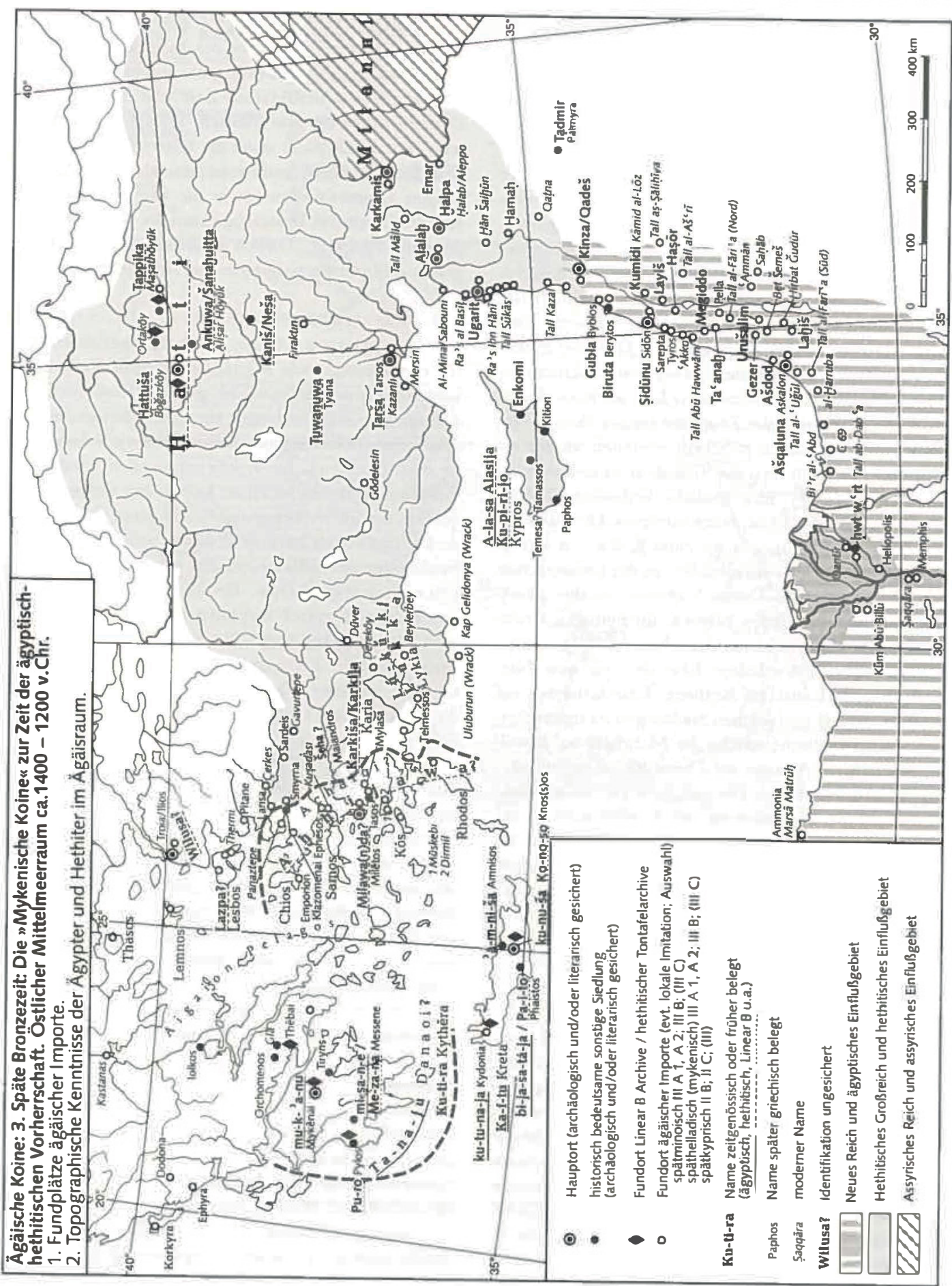

der min. Palastära zusammen: Schon im I s.Jh. v. Chr. fielen die min. Siedlungen auf den Kykladen in myk. Besitz, und spätestens um I 400 gerieten Knosos und der Großteil Kretas unter myk. Herrschaft. In der Folge ging das gesamte Kommunikations- und Handelsnetz
Kretas im östl. Mittelmeer an die Mykener über, und min. Kolomen in der Ägäis und in Kleinasien (z.B. $\rightarrow$ Rhodos, $\rightarrow$ Milet) wurden mykenisch. In ihrem Aufstieg zur Führungsmacht in der Ägäis und in der geogr. Weite ihrer Außenbeziehungen (s.u.) war die 
myk. Palastkultur das Erbe der min. Koine. Verbunden mit diesen Vorgängen war die Akkumulierung von Reichtum an den palatialen myk. Zentren.

Myk. Paläste sind bisher für die Argolis (Mykene, Tiryns, Midea), Böotien (Theben, Orchomenos), für Messenien (Pylos) und für Kreta (Knosos) bezeugt. Ob die Großanlage im böotischen Gla und der Gebrauch der Linear B-Schrift im westkret. Chania ("Kydonia") diese beiden Fundorte als Paläste ausweisen, wird debattiert. Für Lakonien, Attika (Athen, Eleusis) und die Gegend um Volos ("Iolkos") werden Paläste postuliert, sind aber arch. nicht gesichert. Arch. Quellen und Linear B-Texte bezeugen die erstaunliche Einheitlichkeit, welche die Paläste in Architektur, Organisation, Kult und Religion, Wirtschaft und Kunst an den Tag legten. Titel (uranax) und Charakter des Königtums, Plan und Ausstattung der Königshalle ("Megaron"), Aufbau und Titel der Würdenträger-Hierarchie und offizieller Kult waren an allen Palastzentren gleich und belegen dieselbe Staatsform und dieselbe Königsideologie. Diese Einheitlichkeit ließ in der Forsch. gelegentlich die Vorstellung eines gesamtgriech. "Mykenischen Reiches" unter der Führung Mykenes entstehen. Die Linear B-Texte unterstützen dies aber nicht. Sollte daher der hethit. Ländername $\rightarrow$ Achijawa tatsächlich auf das griech. Festland zu beziehen sein, dann nicht im Sinne eines gesamtmyk. Reiches. Wohl aber weisen Sachkultur und Linear B-Texte auf enge Beziehungen der Palastzentren untereinander im 14.Jh. und im größeren Teil des 13. Jh. v. Chr. hin. Sie verliefen auf diplomatischer, polit.wirtschaftlicher, kultureller und gelegentlich mil. Ebene. Die homogene myk. Kultur zw. ca. 1400 und I $250 / 30$ v. Chr. wurde demnach von Palästen geschaffen und getragen. Sie strahlte aus in jene Regionen des myk. Griechenland, wo es keine Paläste gab. Herrschaftsgebiete nachgeordneten Ranges, myk. "Provinzen", orientierten sich dort am Vorbild der Palastkultur. Sie übernahmen spirituelle Schöpfungen z.B. der rel. Sphäre (Phi-, Tau-, Psi-Idole, Rinderfigürchen, Kultgefäße) und des Totenkultes (Grabarchitektur, Grabsitten), doch auch Architekturelemente, Hausformen, Lebensstil. Ebenso wurden die technologischen Errungenschaften und die Stilrichtungen der Palastwerkstätten auf den Gebieten von Töpferei und Koroplastik, Bronzehandwerk, Goldschmiedekunst, Holz-, Stein- und (Elfen-)Beinverarbeitung in den Prov. rasch aufgenommen. Originalerzeugnisse palatialer Werkstätten erreichten die Prov. durch Handel und diplomatischen Verkehr. Auch Rohmaterialien, die aus dem von den Palästen dominierten Außenhandel stammten, erreichten die Prov.regionen erst sekundär. Meisterleistungen der myk. Koine wie monumentale und repräsentative Architektur, Wand- und Fußbodenmalerei und namentlich Fresken, Luxusobjekte exklusiven Kunsthandwerks blieben den Palästen vorbehalten. Regionale Varianten der Koine entstanden z.B. auf $\rightarrow$ Kreta und Rhodos, wo die min. Tradition noch stark verwurzelt war.
Während der Palastära nahm der myk. Einfluß im gesamten Mittelmeerraum zu. Kreta, die ägäischen Inseln, Phokis, das Spercheiostal und Westthessalien wurden Teil der myk. Koine, die auch in die Gebiete nördl. des Olymp vordrang. An der anatolischen Küste entstanden myk. Siedlungen (z. B. Milet, lasos, Panaztepe) und auch Troia weist Spuren myk. Präsenz auf. Im Westen siedelten sich Mykener im Golf von Tarent und auf Sizilien an, während Zypern zwar unter starken myk. Einfluß geriet, aber keine myk. Präsenz jenseits individueller Ansiedlungen aufweist. Die Eingliederung dieser Gebiete in das Einflußgebiet der myk. Koine erfolgte auf Initiative der Paläste, denen sie als Stützpunkte für die Handelsverbindungen und den diplomatischen Verkehr mit Ägypten, der Levante (Ras Schamra-Ugarit), nach Anatolien, nach Norden und ins westl. Mittelmeer dienten. Oriental. und myk. Texte, Bildzeugnisse und Sachobjekte bezeugen vor allem den Osthandel, der seinen Höhepunkt in der Periode von ca. I 340 v. Chr. (Echnaton und die Residenz in Tell Amarna) bis ca. I 250 (Ramses II.) erlangte. Myk. Objekte (meist Vasen) kamen damals bis ins Innere Anatoliens, weit ins syr. Hinterland und nach Mittel- und Oberägypten. Auf myk. Seite wurde dieser vorwiegend mit Metallen, Öl, Wein, Getreide und Sachgütern (bis zur Luxusklasse) befaßte Handel von den Palästen monopolisiert und erreichte die Prov.regionen nur sekundär. Umgekehrt stammten die im Orient und im Westen gefundenen myk. Objekte aus den palatialen Zentren.

Gegen Ende des 13. Jhs.v. Chr. (um 1250/30) begann sich die myk. Koine in regionale Sonderentwicklungen aufzulösen. Die Paläste gerieten damals in eine Situation großer Bedrängnis durch Ursachen, die hier nicht erörtert werden müssen; infolgedessen wurden anscheinend auch die überregionalen Kommunikationsstrukturen unterbrochen. Mit dem Untergang der myk. Palast-Staaten und ihrer Kultur am Ende des 13. Jh. v. Chr. fand auch die myk. Koine ihr Ende. Auf sie folgte der Regionalismus der nachpalatialen Zeit (12./11.Jh. v. Chr.).

S. D.-J.

1]. F. Cherry, in: Proceedings of the Prehistoric Society 47, 198 1, 41-68 2 M. H.Jameson, C. N. Runnels, T. H. van Andel, A Greek Countryside: The Southem Argolid from Prehistory to the Present Day, 1994, 341-342 3 C. RENFRew, in: AJA 71, 1967, 1-20 4 Ders., The Emergence of Civilisation: The Cyclades and the Aegean in the Third Millenum B.C., 1972, 308-338; 356-358

5 C. Broodbank, in: AJA 93, 1989, 319-337 6 Ders., in: World Archaeology 3, 1993, $315-3317$ H.SchlirmanN, Bericht über die Ausgrabungen in Troja in den Jahren 1871 1873, 295-301. Taf. 192-209 8 C. Reinholdt, in: JDAI 108, 1993, 1-41 9 J. S. Soles, The Prepalatial Cemeteries at Mochlos and Goumia and the House Tombs of Bronze Age Crete, 1992, 255-258 10 N.H. Gale, Z.A. Stos-Gall, in: J.A. MacGillivray, R. L.N. Barber (Hrsg.), The Prehistoric Cyclades, 1984, 255-276 11 Z. A. Stos-Gale, N. H.Gale, G.R. Gilmore, in: Oxford Journal of Archaeology 3, 1984, 23-43 12 Z. A. Stos-Galc, in: H. Hauptmann, E. Pernicka, G.A. Wagner (Hrsg.), Old 
World Archaeometallurgy, Der Anschnitt, Beih. 7, 1989 , 279-292 13 M. 13. Cosmoroulos, The Early Jronze 2 in the Aegean, I991, 56-73 14 P. Ferioli, E. Fiandra in: I. PINI (Hrsg.), Fragen und Probleme der brz.zettlichen ägäischen Glyptik, Corpus der mun. und myk. Siegel, Beil. 3, 1989, 41-53; 15 Dies. 111: T. G. Palaima (Hrsg.), Aegean Seals, Sealings and Adninstration $=$ Aegaeum $5,1989,221-229$ 16 O. Н. KRYSZKOWSKA, 111: I3SA 83, 1988, 209-234 17 Dies., in: I. PINI (Hrsg.), Fragen und Probleme der bronzezeithchen ägisischen Glyptık, 1989, 1 1 1-126 18 P. M. WARren, in: Pepragmena tou 4 . Kretologikou Synedriou, 198 I, 628-637 19 W. DörPFEld, Alt-Ithaka, I 927 20 K. Branigan, in: I3SA 70, 1975, 37-49 21 13. GovedariCA, in: D. SRe jovic, N. TASiC (Hrsg.), Hiigelbestattungen in der Karpaten-Donau-Balkan-Zone während der aneolithischen Epoche, 1985, 57-70 22 O. HöcкMANN, 111: Kunst und Kultur der Kykladeninseln in 3. Jt. v. Chr., 1976, 168-175 23 J. L. DAvis, in: AJA 96, 1992, 754

24 J. B. RutTer, in: AJA 97, 1993, 764-767 25 J. F. Cherry, in: C. Renfrew, J. F. Cherry (Hrsg.), Peer Polity Interaction and Socio-Political Change, 1986, 19-45 $26 \mathrm{~L}$. BASCH, Le musée imaginaire de la marine antique, 1987, 93-115 27 D.MAtSAS, 11: Studia Troica I, 1991, I 59-179 28 W.-D. Niemeier, 11]: M. Marazzi, S. Tusa, L. VAGNETTI (Hrsg.), Traffici micenei nel mediterranea: Problemi storic1 e documentazione archeologica, 1986, 245-247 mit Abb. 429 Z.A.Stos Gale, C. F. MacDonald m: N. H. Gale (Hrsg.), Bronze Age Trade in the

Mediterranean, 1991, 254, Abb. 130 G. W Alberc, Ägypten und Levante 2, 1991, 111-118 31 M. H. WIENER, in: Thera and the Aegean World III.1, 1990, 1 50-1 5132 S. Hood, in: R. HäGG, N. Marinatos (Hrsg.), The Minoan Thalassocracy: Myth and Reality, 1984, 33-37 33 F. SchacherMEYr in: Thera and the Aegean World I, 1978, 423-428 34 T. G. Palaima in: Temple University Aegean Symposium 7, 1982, 15-22 35 E.B. FreNCH, Archaeological Reports 40, 1994, $6936 \mathrm{E}$. und Y.Sakellarakis, in: R. HäGg, N. Marinatos (Hisg.), The Minoan Thalassocracy: Myth and Reality, 1984, 201-202 37 C. G. Starr, Historia 3, 1954/55, 282-294 38 G. HuxLEY, Minoans in Greek Sources, 1968, 2-3

39 W.-D. Nicmeier in: Aux origines de l'hellénisme: La Crête at la Grèce, Hommage à H. v. EFFEnT ERRE, I984, I I I-II 40 W.-D. Niemeicr in: S. Gitin (Hrsg.), Recent Excavations in Israel: A View to the West, Archaeological Institute of America, Colloquia \& Conference Papers No. I, 1995, 1-15 41 M. BietaK in: W. V. Davirs, L. SCIIOField (Hrsg.), Egypt, the Aegean and the Levant: Interconnections in the Second Millenium B. C., 1995, 19-28.

H.-G. Bucinholz, V. Karageorguis, Altägäis und Altkypros, $1971 \cdot \mathrm{J}$. Chadwick, Die myk. Welt, $1979 \cdot$ E. H. Cline, Sailing the Wine-Dark Sea. International Trade in the Late Bronze Age Aegean, $1994 \cdot$ S. Hiller, O. PAnagl, Die frülggriech. Texte aus myk. Zeit, ${ }^{2} 1986 \cdot$ K. KILIAN, The Emergence of the ivanax Ideology in the Mycenaean Palaces, in: Oxford Journal of Archaeology 7, 1988, 291-302 - F. SChaChermeyr, Die ägäische

Frülızeit II, $1979 \cdot$ R.-D. Treuil, J.C. Pascai.-Poursat, G. Touchass, Les civilisations égéennes du neolithique et de l'âge du bronze, 1989.

KARTENLITERATUR:

H.-G. Buchнolz (Hrsg.), Ägüische Bronzezeit, $1987^{\circ}$ G. Bunnens, A. Kusciike, W. Röllig, Palästina und Syrien zur Zeit der ägyptisch-hethitischen Vorherrschaft, TAVO
B III 3, - E. Edel, Die Ortsnamenhste aus dem

Totentempel Amenophis III., 1966, $33 \mathrm{ff}$.

Neubearbeitung - M. Forlani, Kleinasien. Das

Hethitische Reich im 14.-13. Jahrhundert v. Chr. TAVO

13 111 6, 1992 - F. GomaÁ, R. Hannig, Ägypten zur Zeit des Neuen Reiches, TAVO B III I, 1991 - G. KорСKE, Handel, ArchHom Kap. M, 1990 - G. A. LeHMANN,

Die mykenisch-frühgriechische Welt und der östhche Mittelmeerraum in der Zeit der Seevölkennvasionen, $1984 \cdot$ J. Osing in: JEA 68, 1982,77f.

Aegidius. Gallischer Aristokrat, von Kaiser $\rightarrow$ Maiorianus 456 oder $457 \mathrm{n}$. Chr. zum comes et magister utriusque militiae per Gallias ernannt, verlor Köln an die Franken, entriß 458 den Burgundern Lyon und verteidigte Arles gegen die Westgoten, die er $46_{3}$ im Bunde mit dem Salierkönig Childerich bei Orléans besiegte. $46 \mathrm{I}$ erkannte er Maiorianus' Nachfolger nicht an und schuf sich in Nordgallien mit Zentrum Soissons eine unabhängige Machtstellung, die er bis zu seinem Tode 464/65 vor allem gegen $\rightarrow$ Ricimer behauptete und seinem Sohn Syagrius hinterließ (Prisc. fr. 30; Greg. Tur. Franc. 2,1 1; 1 2; 18; 27; Chron. min. 2, 33 MommSEN). PLRE 2, 11-13.

A.DemandT, s.v. magister militum, RE Suppl. 12 , $687 \rightarrow 1$.

K.PJ.

\section{Ägineten s. Bauplastik}

\section{Äginetischer Standard s. Münzfïße}

Aegritudo Perdicae. In Codex Harleianus 3685 (I 5. Jh.) überliefertes Epyllion (250 Hexameter), in dem die unselige Liebe des nach dem Studium in Athen heimgekehrten P. zu seiner Mutter mit beachtlichem Einfiihlungsvernögen als psychische Erkrankung behandelt wird (s. 174). Ursache ist ein versäumtes Opfer an Venus. P. überwindet die Rachsucht der Göttin und Amors, indem er sich zum Selbstmord entschließt, um so auch den in ihm wütenden Liebesgott zu vernichten. Der Stoff dürfite einer hell. Quelle entstammen (vgl. Ps.-Soran. vita Hippocr. 3; Claud. carnı. min. 8; Drac. Romulea 2,39-42, Anth. Lat. 220) und in der Umgebung des $\rightarrow$ Dracontius versifiziert worden sein. Ob dieser selbst der Verf. ist [3. I644], bleibt ungewiß [5].

Ed.: 1 F. Vollmer, PLM² S, 238-250 2 L.ZurLI, 1987. Lit .: 3 F. Vollmer, Dracontius [4], in: RE 5, I635-1644 4 J.M. Hunt, The A. P., Diss. Bryn Mawr 19705 É. WolfF, L'A. P., un poème de Dracontius?, in: RPh 62, 1988, 79-89.

K. SM

Ägypten. A. Einleitung B. Prähistorie Und Staatsentstehung C. Erste Zwischenzeit D. Zweite Zwischenzeit E. Spätzeit

\section{A. Einleitung}

Das Land am Nil vom I. Katarakt bis zum Mittelmeer, ägypt. Km.t "das schwarze (Land)", griech. 